\title{
Die Romantrilogie des argentinisch-österreichischen Schriftstellers Germán Kratochwil: das Große im Kleinen Patagoniens
}

\author{
Reinhard Andress \\ Loyola University Chicago, randress@luc.edu
}

Follow this and additional works at: https://ecommons.luc.edu/modernlang_facpubs

Part of the Modern Languages Commons, and the Modern Literature Commons

\section{Recommended Citation \\ Andress, Reinhard. Die Romantrilogie des argentinisch-österreichischen Schriftstellers Germán Kratochwil: das Große im Kleinen Patagoniens. Revista de Filología Alemana, 27, : 81-96, 2019. Retrieved from Loyola eCommons, Modern Languages and Literatures: Faculty Publications and Other Works, http://dx.doi.org/10.5209/rfal.64352}

This Article is brought to you for free and open access by the Faculty Publications and Other Works by Department at Loyola eCommons. It has been accepted for inclusion in Modern Languages and Literatures: Faculty Publications and Other Works by an authorized administrator of Loyola eCommons. For more information, please contact ecommons@luc.edu. cc) (i) $\Theta$

This work is licensed under a Creative Commons Attribution-Noncommercial-No Derivative Works 3.0 License. (c) Universidad Complutense de Madrid, 2019. 
Revista de Filología Alemana

ISSN: 1133-0406

https://dx.doi.org/10.5209/rfal.64352

\title{
Die Romantrilogie des argentinisch-österreichischen Schriftstellers Germán Kratochwil: das Große im Kleinen Patagoniens
}

Reinhard Andress ${ }^{1}$

Recibido: 4 de abril de 2018 / Aceptado: 24 de mayo de 2018

Zusammenfassung. Der 1938 in Österreich geborene Germán Kratochwil emigrierte früh nach Argentinien, kam aber erst spät zur Literatur nach einer langen Karriere als Sozialwissenschaftler. Inzwischen sind drei Romane auf Deutsch erschienen: Scherbengericht (2012), Río Puro (2013) und Territorium (2016). Da der Handlungsort aller drei Werke Patagonien bleibt, könnte man von einer patagonischen Romantrilogie des Autors sprechen. Anhand eines beträchtlichen Figurenarsenals liegen die motivischen Konstanten in Auseinandersetzungen mit Vergangenheit und Gegenwart, gesellschaftlichen Ausstiegsversuchen und im Hineinwirken von Weltkonflikten ins nur scheinbar abgeschiedene Patagonien. In einer genauen Textanalyse werden die genannten Motive herausgearbeitet. Letztendlich zeigt sich im Kleinen Patagoniens das große Weltgeschehen, wobei sich eine schwebende Weltanschauung ergibt, die auf eine wenig gesicherte Zukunft weist.

Schlüsselwörter: Kratochwil; Deutschsprachige Literatur; Argentinisch-österreichische Literatur; Patagonien.

\section{[en] The Trilogy of Novels by the Argentinian-Austrian Writer Germán Kratowchwil: the Big World in the Small World of Patagonia}

\begin{abstract}
Germán Kratochwil, born 1938 in Austria, emigrated early on to Argentina but didn't come to literature until later in life after a long career as a social scientist. Meanwhile, he has published three novels in German: Scherbengericht (2012), Río Puro (2013) und Territorium (2016). Since the setting of all three works is Patagonia, it makes sense to speak of a Patagonian trilogy of novels by the author. On the basis of a considerable arsenal of characters, the motifs lie in confrontations with the past and present, attempts to escape from society, and the effects of world conflicts on an only seemingly isolated Patagonia. These motifs are explored in an exact textual analysis. Ultimately, the events of big world show themselves in the small world of Patagonia, resulting in a worldview that points to a hardly secure future. Keywords: Kratochwil; German Literature; Argentinian-Austrian Literature; Patagonia.
\end{abstract}

\section{[es] La trilogía de novelas del escritor argentino-austriaco Germán Kratochwil: lo grande en lo pequeño de la Patagonia}

Resumen. Germán Kratochwil, nacido en Austria, en 1938, emigró pronto a Argentina, pero llegó tarde a la literatura tras una larga carrera como sociólogo. Entretanto han aparecido tres novelas en alemán: Scherbengericht (2012), Río Puro (2013) y Territorium (2016). Como el escenario de las tres es la Patagonia, podría hablarse de una trilogía de novelas patagónicas. De la mano de un considerable arsenal

Loyola University Chicago (USA)

E-Mail: randress@luc.edu 
de personajes, los motivos se mantienen como constantes en la confrontación entre pasado y presente, en los intentos de ascenso social y en los efectos de los conflictos internacionales en una Patagonia alejada del mundo tan solo aparentemente. A través de un análisis textual preciso se estudian aquí los motivos mencionados para concluir que en la pequeñez de la Patagonia, aun con una visión del mundo un tanto oscilante, los grandes acontecimientos del mundo apuntan a un futuro poco cierto.

Palabras clave: Kratochwil; Literatura en lengua alemana; Literatura austríaco-argentina; Patagonia.

Inhaltsverzeichnis. 1. Scherbengericht: gescheiterte Auseinandersetzung mit Vergangenheit und Gegenwart. 2. Río Puro: Ausstiegsversuche. 3. Territorium: Einbruch der großen Welt in Patagonien. 4. Schlussbetrachtung.

Cómo citar: Andress, R., «Die Romantrilogie des argentinisch-österreichischen Schriftstellers Germán Kratochwil: das Große im Kleinen Patagoniens», Revista de Filología Alemana 27 (2019), 81-95.

Der 2012 erschienene Roman Scherbengericht war Germán Kratochwils Erstlingswerk. Doch dreht es sich dabei nicht um einen Autor jüngeren Alters, denn er wurde 1938 in Korneuburg (Niederösterreich) geboren. Nach dem Krieg emigrierte die Familie nach Argentinien, wo Kratochwil in Buenos Aires, später in München, Berlin und Hamburg studierte und 1973 als Sozialwissenschaftler promovierte. Beruflich machte er sich einen Namen vor allem als Büroleiter der Internationalen Organisation für Migration in Peru, Paraguay und Uruguay. Heute lebt er sowohl in Buenos Aires als auch in Patagonien. Er kam also spät zur Literatur, und zwar mit relativem Erfolg, denn in seinem Erscheinungsjahr landete Scherbengericht auf der „Longlist“ des Deutschen Buchpreises. ${ }^{2}$ Kratochwil schob dann zwei weitere Romane nach: Río Puro (2013) und Territorium (2016). ${ }^{3}$

Da der Handlungsort aller drei Werke Patagonien bleibt, könnte man von einer patagonischen Romantrilogie des Autors sprechen. Anhand eines beträchtlichen Figurenarsenals, das viele Einwanderergeschichten enthält, liegen die motivischen Konstanten der Trilogie in Auseinandersetzungen mit Vergangenheit und Gegenwart, gesellschaftlichen Ausstiegsversuchen und im Hineinwirken von Weltkonflikten ins nur scheinbar abgeschiedene Patagonien. In einer genauen Textanalyse sollen im Folgenden die genannten Motive herausgearbeitet werden. Letztendlich zeigt sich im Kleinen Patagoniens das große Weltgeschehen, wobei sich eine schwebende Weltanschauung ergibt, die auf eine wenig gesicherte Zukunft weist.

\section{Scherbengericht: gescheiterte Auseinandersetzung mit Vergangenheit und Gegenwart}

Der erzählerische Anlass vom Scherbengericht ist der 90. Geburtstag der österreichischen Auswanderin Clementine, der am 1. Januar 2000 zusammen mit Freunden und Verwandten aus drei Generationen auf einem Gutshof in der Nähe vom patagoni-

2 Vgl. www.deutscher-buchpreis.de/de/544785/. [01.04.2018]. Vom Roman erschien auch 2013 eine Fischer Taschenbuchausgabe.

3 Abgesehen von den Romanen liegt auch eine Erzählung von Kratochwil mit dem Titel „Strandgut“ vor, erschienen in Dorothea Löcker und Alexander Potyka (Hrsg.), Mordserfolg. Sechzehn Kurzkrimis (2014). Wie der Titel des Erzählbandes andeutet, handelt es sich um eine Kriminalgeschichte, die in diesem Falle in Montevideo spielt. 
schen Quemquemtréu, einem erfundenen Ort, gefeiert werden soll. Es ist „ein dreifaches Zeitspannen-Scharnier“, wie es eine der Figuren zum Ausdruck bringt: „Ein Jahr gehe seinem Ende zu, ein neues breche an, ein Jahrhundert laufe aus, ein neues stehe bevor, ein Jahrtausend sei vergangen und ,freudetrunken' betrete man das nächste. Man hüpfe über drei Zeitmarken hinweg in ein neues Epochengefüge, das vom ersten Jänner 2000 an gelte“ (Kratochwil 2012: 160-161). Es ist ,[e]in verdichteter Moment Zeit"“ (Kratochwil 2012: 161), der den Roman ebenfalls zu einer Verdichtung der Zeit werden lässt. Durch in verschiedene Figuren aufgefächerte Rückblenden wird Vergangenheit in die Gegenwart der Geburtstagsfeier gezogen, um jene zu verarbeiten und diese mit einem Blick in die Zukunft zu betrachten. In seiner Rezension schreibt Dirk Knipphals treffend: ,Jede Erzählperspektive hat ihre eigene Sprachmelodie und Erzählfarbe, in technisch einleuchtend inszenierten Rückblenden wird dabei von hin und hergeworfenen Schicksalen berichtet" (Knipphals 2012). Dabei scheitert die Auseinandersetzung mit Vergangenheit und Gegenwart, was eine unsichere Zukunft über den unmittelbaren Kontext des Romans hinaus andeutet.

Dem sozialwissenschaftlichen Autor steht die Figur des Entwicklungshelfers Martin Holberg sicher am nächsten. Der Tod seiner Frau Judith hat ihn und seine zwei Kinder in eine Lebenskrise gestürzt, die sich für ihn als Midlife-Crisis auch beruflich auswirkt. Mit der Tochter Katha auf dem Wege zur Geburtstagfeier muss er als Vertreter der Stiftung „Boden und Frieden“ versuchen, eine Mapuche-Gemeinde von den Vorteilen eines Stausees zu überzeugen. Es ist eine Arbeit, an deren „offizielle Geseire“ (Kratochwil 2012: 21) er ermüdet nicht mehr glaubt. Seine „,nagenden Zweifel“ führen ihn zu Fragen: „Gibt es denn irgendwo noch festen Boden für einen einfältig und barfüßig gewordenen Sozialwissenschaftler? Wohin führt mich - wenn ich denn einen haben sollte - mein Weg?" (Kratochwil 2012: 21). Zusammen mit der Tochter Katha, die auch schlecht mit dem Tod der Mutter zurechtkommt und gerade aus der Psychiatrie einer Klinik entlassen worden ist, will er sich aufs Land, möglicherweise auf den Gasthof, wo die Geburtstagsfeier stattfindet, zurückziehen. Damit wollen sie zu einer Vergangenheitsbewältigung ansetzen, die zu einem psychischen Erneuerungsversuch führen soll, was sich als vergeblich zeigen wird.

Der jüngere Sohn Gabriel ist vor zwei Jahren von zu Hause abgehauen, um sich vom starken Vorbild des Vaters zu befreien. Tief in Patagonien hat er Zuflucht im sogenannten Schalerbund, einer Sekte, gefunden, wo er den Vater-Komplex überwinden will. Bei der Geburtstagsfeier kommt es zur ersten Begegnung mit dem Vater seit dem Weggang von zu Hause. An soziale Harmonie glaubt Gabriel nicht mehr und regt sich über diejenigen auf, „,ie mit der Lügenpredigt von der Gleichheit aller Menschen einen Notausgang für das friedliche Zusammenleben der Erdenbürger beschwören und uns glauben machen wollen, ein harmonisches Miteinander aller Geborenen sei möglich“ (Kratochwil 2012: 265). Zweifellos ist diese Weltsicht vom Sektenführer Heinz von Futterer beeinflusst, der das anbrechende Jahrhundert so sieht: „Klimakatastrophen, wirtschaftlichen Kollaps, Verelendung, Massenmigration, Mauern und Stacheldraht zwischen Erster und Dritter Welt, heimtückische Pandemien, Amokläufe und Explosionen sozialer Gewalt, mörderischer Terrorismus und am Ende, mit zwingender Konsequenz, den Atomkrieg“" (Kratochwil 2012: 178). Paragleitend auf dem Wege zur Großmutter fragt sich Gabriel vieldeutig: „Verdammt, ich hänge in der Luft, wo soll ich landen?"“(Kratochwil 2012: 201).

Der biographische Hintergrund der Großmutter Clementine ist eine österreichische Auswanderergeschichte während der Dollfuß-Zeit. Inzwischen verwitwet, be- 
dingen ihr hohes Alter und die Überzeugung, es sei ihr letzter Geburtstag, die intensive Beschäftigung mit der Vergangenheit. Was bei inneren Monologen und der Geburtstagsfeier zum Vorschein kommt, sind weitgehend unverarbeitete antisemitische, ausländerfeindliche und homophobische Ressentiments. Angesichts der Schwierigkeiten der beiden Enkelkinder und ihrer jüdisch-katholischen Eltern sind für sie „Mischehen [...] unbedingt zu vermeiden“: „An solchem Mischmasch wird die moderne Welt zugrunde gehen, zuerst die USA, dann Österreich - wir haben es ja schon einmal erlebt. Jetzt kommen die Slawen wieder, die Türken, die Serben, die Asiaten, sogar die Afrikaner; Juden gibt es ja kaum noch in Österreich. Ob die nicht doch das kleinere Übel gewesen wären?" (Kratochwil 2012: 267). Aus Clementines Sicht ist das Jahrhundert „vergiftet“ (Kratochwil 2012: 91), wobei sie nicht sieht, wie sehr ihre unbewältigte Vergangenheit zu dieser Vergiftung beiträgt.

Der Gasthof wird von Treugott Lagler - Trigo genannt - und seiner Frau Rotraut betrieben, die ebenfalls mit Auswanderergeschichten verbunden sind. Vor achtzig Jahren hatte Treugotts Vater, ein Bauernknecht aus Südtirol, das Stück Land in Patagonien im Rahmen der argentinischen Siedlungspolitik geschenkt bekommen, es mit Mühe aufgebaut, schließlich dem Sohn zusammen mit einem inzwischen in die Höhe gewachsenen Lindenbaum überlassen, ,gedacht als Stammessymbol künftiger Lagler-Geschlechter" (Kratochwil 2012: 41). Mit einem verkürzten Bein geboren hat sich aber dieses im Laufe der Jahre immer mehr als körperliche Belastung für Treugott erwiesen, abgesehen von der „Missgeburt“ (Kratochwil 2012: 49) des scheinbar autistischen Sohnes Quique, der ein unverbesserlicher Tierquäler ist. Bei Treugott war die Vergangenheit weitgehend positiv; es ist die Gegenwart, die ihm große psychische Schwierigkeiten bereitet, und er spürt eine „Zunehmende Verbitterung“ (Kratochwil 2012: 130). Besonders Rotraud ist um ihren Mann besorgt. Über eine Agentur hatte Treugott sie als Frau nach Argentinien geholt, sie dabei als nordmährisches Flüchtlingskind aus ihrer Bochumer Langeweile und Aussichtslosigkeit gerettet. Sie kümmert sich rührend um alle und steckt als Köchin und Bäckerin hinter dem reichlichen Festessen der Geburtstagsfeier. Doch auch bei ihr stellt der missratene Sohn die geglückte Auswanderung in Frage: ,Wer soll diesen Hof dereinst weiterführen?" (Kratochwil 2012: 124). Es zeigt sich keine harmonische Zukunft.

Weitere Geburtstagsgäste sind Gretl und Elias Königsberg, beide Juden, die der argentinische Konsul in Wien, Martins Großvater, in sein Land gerettet hatte. Elias ist Psychologe und versucht, bei den Urlaubsaufenthalten auf dem Gutshof seine Erinnerungen aufzuschreiben. Eine positive Veränderung der Welt stellt er in Frage: „Ich muss gestehen, dass mir das Einrichten eines vermeintlichen vernünftigen und vernunftsuchenden Umgangs zwischen den Menschen immer schwerer fällt" (Kratochwil 2012: 280-281). Dennoch ist aus seiner Sicht das bunte Gemisch von Menschen bei der Geburtstagsfeier beachtenswert:

[...] dieses Sich-zusammen-Ertragen sei die letzte Möglichkeit für unsere Generation, die Verirrungen des Jahrhunderts friedlich auszuleben (von ,bewältigen “ natürlich keine Rede). Wirklich verändern könnten wir uns eh nicht mehr; und wir hier oben, am Ende unserer Lebenswege, verwirklichten im Mikrokosmos des Tilo-Hofes recht eigentlich eine bewundernswerte Utopie (Kratochwil 2012: 209).

Wie hier deutlich wird, gibt es für Elias keine Vergangenheitsbewältigung an sich; gegenseitige Toleranz ist das Beste, was man sich erhoffen kann. Das fällt Gretl wie- 
derum sehr schwer, denn einer der Gäste ist Siegmund Rohr, ein ehemaliger Nazi, der aus Mauthausen mit dem Tod ihres Sohnes Moritz im dortigen KZ zusammenhängen könnte: „Wie ungerecht unsere doppelte Last: zuerst Vertreibung aus der Heimat oder Tod, dann Heimsuchung durch die Täter" (Kratochwil 2012: 216).

In der Tat kommt Rohr aus Mauthausen, wenn er auch scheinbar nicht im KZ tätig war, vielmehr als Obergefreiter hinter der Ostfront diente. Dennoch hat er sich als ehemaliger Nazi auf Schleichwegen nach Argentinien abgesetzt. ${ }^{4}$ Von Katha und Martin wird er die letzte Strecke zur Geburtstagsfeier mitgenommen, wobei es zur Begegnung mit Nicko kommt, der in einem Wahnsinn durch die patagonische Landschaft irrt. Hier wird Roths unbewältigte Nazi-Vergangenheit deutlich, denn er stellt sich vor, „wie er mit der rechten Hand, bei ausgestrecktem Zeigefinger und aufgerichtetem Daumen, auf den Scheißer zielte, abdrückte, mehrmals, peng, peng, peng ... " (Kratochwil 2012: 235). Unverfroren erkennt Katha seine stark diskriminierende Haltung: „Also ein minderwertiges Element, dieser Nicko? Klasse, Roth, für ihre einundneunzig Jahre sind Sie ja noch ganz gut drauf! Sind Sie etwa der Geliebte meiner Oma?" (Kratochwil 2012: 241).

Treugotts Selbstmord wird schließlich der unbeabsichtigte Höhepunkt der Geburtstagsfeier. Der Rollstuhl, den Rotraud ihrem Mann besorgt und bei der Feier übergibt, scheint das Bewusstsein seiner körperlichen Hilflosigkeit nur noch stärker hervorzukehren. Er erhängt sich im Stall, was Königbergs Sicht auf den Hof mit dem Lindenbaum als Ort der Utopie stark hinterfragt. In dem Kontext kann das dem Roman vorangestellte Zitat aus Adalbert Stifters Bildungsroman Nachsommer (1857) nur ironisch verstanden werden: „Die Linde ist der Baum der Wohnlichkeit“ (Kratochwil 2012: 7). Einen „Scherbenhaufen“ der mangelnden Vergangenheitsbewältigung und Lebensfähigkeit in der Gegenwart könnte man meinen, wenn der Roman nicht „Scherbengericht“" hieße, d.h. den Namen jenes Verfahrens im alten Griechenland trägt, bei dem Menschen verbannt werden konnten, indem man ihre Namen auf Scherben schrieb, die ausgezählt wurden, heute in folgender Bedeutung: „,mit jemandem hart ins Gericht gehen". ${ }^{5}$ So gesehen ist es vielleicht der Autor, der in den Worten Martins über die „Unerwünschten, Zwiespältigen, Diskriminierern und Diskriminierten, Ausgewanderten, Ausgewiesenen, Ausgegrenzten, Vetriebenen [sic!], Verschollenen, Verschütteten, Untergetauchten“" (Kratochwil 2012: 310) des Romans ein Scherbengericht hält. „Ist irgendwann Schluss damit?“ (Kratochwil 2012: 310) fragt Martin dann auch und impliziert, was im 21. Jahrhundert werden soll, wenn schon das 20. Jahrhundert nur schwer mit Vergangenheit und Gegenwart zurecht kommt.

Auch im Zusammenhang mit dieser Frage kann man Martin zitieren, der, wie anfangs erwähnt, Kratochwil am nächsten zu stehen scheint und dessen Aussagen auch deshalb eine besondere Bedeutung beizumessen ist, weil die Eingangs- und

4 Roth steht hier stellverstretend für die nicht unbeträchtliche Anzahl von Nazis, die sich nach Argentinien absetzen konnten und von denen Adolf Eichmann der prominenteste war. Josef Mengele und Erich Priebke fanden den Weg nach Patagonien. Vgl. Holgar Medings Flucht von Nürnberg (1998) oder Uki Goñi: The Real Odessa: How Peron Brought the Nazi War Criminals to Argentina (2002), 2006 auf Deutsch als Odessa: Die wahre Geschichte. Fluchthilfe für NS-Kriegsverbrecher. Vgl. ebenfalls Frank Garbelys Evitas Geheimnis (2003), Gerald Steinachers Nazis auf der Flucht (2008) oder Bettina Stangneths Eichmann vor Jerusalem (2011).

5 Vgl. „Scherbengericht“ in Wiktionary (http://de.wiktionary.org/wiki/Scherbengericht) oder im Universal-Lexikon (http://universal_lexikon.deacademic.com/118454/Scherbengericht). [01.04.2018] 
Schlusskapitel jeweils aus der Perspektive dieser Figur erzählt werden. Schlaflos nach dem Selbstmord Treugotts tritt Martin vor die Tür und sieht auf der vorletzten Seite des Romans in den ungewöhnlich klaren Sternenhimmel:

Wie verständlich, dass die Menschen sich die Sternbilder ausgedacht haben: Sie vermitteln ihnen so etwas wie Ordnung und Wegzeichen auf ihrer ziel- und grundlosen Reise durch das glitzernde Chaos. Soll das ein Wegkreuz sein, das ins Gelobte Land weist? Den gesegneten Ort, den ich für Katha und mich suche? „Das steht in den Sternen“, wie man so sagt. Nur, hier unten, im patagonischen Tilo-Hof, liegt diese Zuflucht bestimmt nicht. Wie absolut, ja absolut gleichgültig dieses ganze Szenario unseres Umherirrens uns doch umgibt, wie es sich in lichtlosen Tiefen, im Rauschen, im Schimmern und Funkeln, in scheinbaren Wegmarken und Schönheiten doch nur auf sich selbst bezieht! (Kratochwil 2012: 309).

Für Valentin Schönherr ist das ,,ein irritierender Schluss““: „Ob das heissen soll: ,Nun lass mal gut sein, das von früher ist alles nicht mehr wichtig', oder: ,Wir sind für diese Dinge zu alt, das müssen nun andere auf sich nehmen' - das bleibt verstörend offen" (Schönherr 2013). Aus Martins Sicht bleibt das Leben jedenfalls unfassbar, zufällig, geht aber ungeklärt irgendwie weiter. Dabei gibt es auch schöne Momente in ihrer bestechenden Einfachheit, so die „Lust auf ein kräftiges Bauernfrühstück“ (Kratochwil 2012: 311), mit der der Roman im letzten Satz ausklingt. Kratochwil hat ein langes Leben als aktiver Sozialwissenschaftler gehabt, der tief in die große Welt mit ihren vielfältigen Widersprüchen eingetaucht ist und reiche Lebenserfahrungen gesammelt hat, die wiederum in die kleine Welt im Scherbengericht eingeflossen sind. Es ist das Bild eines Lebens, das sich chaotisch und mehr zufällig als rational fortsetzt, dabei stark relativierend, ohne polemische Kanten, ohne Erklärungsmodell, subjektiv, pluralistisch und postmodern.

\section{Río Puro: Ausstiegsversuche}

Scherbengericht spielt sich zwar gegen den Hintergrund von Patagonien ab, doch entwickelt sich die Handlung stark aus der Innenperspektive der vielen Figuren heraus (vgl. Koneffke 2013). In seinem zweiten Roman der Trilogie, Río Puro, verlagert sich die Perspektive auch nach außen. Patagonien selbst wird stärker zu einem zentralen Handlungselement als jener Region des südlichen Südamerikas mit einer Gesamtfläche von über eine Million Quadratkilometer, durch die Anden zwischen Chile und Argentinien aufgeteilt, von steppenartigen Pampas geprägt und dünn von Einwanderern und der indigenen Bevölkerung besiedelt. Schon immer ist es das Ziel von Abenteurern wie dem deutschen Flieger Günther Plüschow (1886-1931) ${ }^{6}$ oder dem englischen Reiseschriftsteller Bruce Chatwin (1940-1989) ${ }^{7}$ gewesen. Zwielichtige Typen wie „Butch Cassidy and the Sundance Kid“, hinter denen Robert Leroy Parker (1866-1908) und

\footnotetext{
6 Vgl. Plüschows Silberkondor über Feuerland (1929), dazu auch die aktualisierte und fortgeschriebene Neuauflage 2007.

7 Vgl. Chatwins Roman In Patagonia (1977), auf Deutsch 1981 als In Patagonien.
} 
Harry Longabaugh (1867-1908) steckten, fanden dort eine Zeitlang eine Bleibe. ${ }^{8}$ Wie schon oben erwähnt, gelangten nach dem Zweiten Weltkrieg auch Nazis nach Patagonien.

Diese historischen Figuren werden alle in Río Puro erwähnt und geben gewissermaßen den Hintergrund für das Thema des Aussteigertums ab, das dem Roman unterliegt und dessen Alternative als Antwort auf die Unwägbarkeiten des Lebens im Scherbengericht gesehen werden kann. An einer Reihe von Figuren probiert Kratochwil die „verhängnisvoll[e] Versuchung namens Patagonien“ (Koneffke 2013) aus; an ihnen spielt er erzählerisch und z.T. mit viel Ironie Ausstiegsversuche durch: deren idealistisch-kulturelle Grundlagen, Naivität, Gefahren, moralische Unterminierung, Scheitern oder auch relativen Erfolg.

Im Roman reist der österreichische, aber in Italien lebende, halb pensionierte Journalist Leopold Kainzer Ende April 2003 nach Buenos Aires, um über die argentinische Präsidentenwahl zu berichten. Dort bittet ihn die Jugendfreundin Livia Melan, ihrem nach Quemquemtréu abgehauenen Ehemann Franz, einem österreichischen, aber seit Jahren in Südamerika ansässigen Experten für Wasserresourcen nachzureisen, um herauszufinden, was sein plötzliches Verschwinden auf sich hat. Die Reise, bei der Kainzer Melan etwa zwei Tage hinterher hinkt, führt in eine Groteske, bei der wir weitere Aussteigerfiguren wie den Oberösterreicher Gregory Hold oder den amerikanischen Millionär Timothy Meyllonet kennen lernen. Gemeinsam haben sie ein ökologisches Interesse am Erhalt der Wasserreserven Patagoniens.

Melans Ausstiegsversuch ist geplant, aber geheim, wobei eine junge uruguayische Assistentin, Maia Morelli, und etwa eine halbe Million Dollar unklare Rollen spielen. Er reist mit dem Geld illegal von der argentinischen auf die chilenische Seite Patagoniens in die Berge hinauf zu Holds Anwesen, und von dort aus soll es weiter zu Meyllonet gehen, der sich hoch oben in den Bergen der Rettung des „reinsten Trinkwasserhaushalte unseres Planeten“ (Kratochwil 2013: 181) widmet. Eventuell will sich Melan mit dem Geld an diesem Projekt beteiligen; Morelli, mit der er sich später in Puerto Montt auf der chilenischen Seite treffen will, soll ihn anscheinend bei seinem Neuanfang begleiten. Mit seinen über sechzig Jahren ist es bei Melan eine verspätete Midlife-Krise wie schon bei Martin im Scherbengericht; er hat erkannt, dass sein Leben ,keine Leihgabe ist, sondern ein einmaliger, eigentümlicher, ausschließlicher, kostbarer, winziger Anteil am Ganzen, am Ewigen“, ein Leben, das „eine neue Richtung braucht“ (Kratochwil 2013: 48): „Darin, und nur darin, sei letztlich vielleicht doch ein Sinn in diesem ganzen Un-Sinn zu erkennen ... im Ausbruch“ (Kratochwil 2013: 93-4).

Dabei ist Melan reichlich mit dem kulturellen Hintergrund seines österreichischen Bildungsbürgertums ausgestattet, der sich vor allem in Zitaten äußert, die das innere und äußere Geschehen einzuordnen versuchen. Er ist auf der Suche nach einem Halt für seine Seele, deren prekären Zustand er mit Hilfe eines Zitats aus Hugo von Hofmannsthals „Gespräch über Gedichte“ (1903) festhält: „Wie der wesenlose Regenbogen spannt sich unsere Seele über den unaufhaltsamen Sturz des Daseins. Wir besitzen unser Selbst nicht“" (Kratochwil 2013: 141). Bei seinem Ausstiegsver-

Vgl. z.B. James D. Horan, Desparate Men: the James Gang and the Wild Bunch (1997). Zum Bekanntheitsgrad dieser Verbrechergeschichte trug die Verfilmung unter dem Titel Butch Cassidy and the Sundance Kid 1969 mit Robert Redford und Paul Newman (Regie: William Goldman) bei. 
such kommt Melan schließlich in der Natur an, wozu dann ein Zitat aus Brochs Tod des Vergil (1945) die Vorlage gibt:

Es war ihm, als hätte er diese Ankunft genauestens vorausgesehen, als handelte er in völligem Einklang mit umfassenden Vorbereitungen. Denn es stand in Brochs Vergil geschrieben: „Bäume umrahmten die Uferstelle, ihre schatten-gesprenkelten Laugassen, landeinwärts leise ansteigend, luden ein, sich ihnen zu nähern, und das Wasser, still zwar in seinem ewigen Spiegel, bespülte den Strand mit hurtig-leisem, weißbesäumtem Auslauf, ein kleines Schäumen zurücklassend, das wie Hörbarkeit im schweigend Unvernehmlichen war, freundlich in dem murmelnden Anfluten, freundlich in dem rieselnden Abbebben." (Kratochwil 2013: 38).

Dass Melan beim Sprung vom Boot den Knöchel verstaucht, ironisiert seinen „mystisch benebelt[en]" Zustand, ein Motiv, das den Roman durchzieht (vgl. Drekonja-Kornat 2013). Letzten Endes erweist sich Melan als ,schwacher Kulturbürger“" (Koneffke 2013), dessen ganze Bildung der rauen Realität Patagoniens nicht standhalten kann. Er macht sich erotische Hoffnungen auf die Mapuche Cintia, die angesichts früherer Misshandlungen Melan ,in einem Anfall rasender Rachsucht“" (Koneffke 2013) vergewaltigt, eine Szene, die weit entfernt ist vom Ausstiegsversuch als Lebensalternative und vom sonstigen, eher ironischen und leichten Erzählton des Romans. Von Meyollonet per Hubschrauber und Flugzeug gerettet, schwebt er am Romanende in Lebensgefahr in einem Krankenhaus in Buenos Aires. Vom Fluss weggeschwemmt und vermutlich unauffindbar ist das Geld. Sein Ausstiegsversuch ist kläglich gescheitert.

Gregory Hold ist episch nicht so vielfältig wie Melan gezeichnet, stellt aber eine weitere Variante eines zunächst einmal gelungenen Ausstiegs dar. Als ehemaliger Schreibwarenvertreter aus dem Innviertel lebt er schon seit Jahren in Patagonien, schreibt für die Jugendzeitschrift Unsere Tierwelt und sitzt an einem Buch mit dem Arbeitstitel „Der patagonische Trinkwasserkrieg“'. Für Melan hat Hold Stifters Naturverbundenheit in die Realität umgesetzt: „Gregor, du hast den Samen des Stifter'schen Naturbekenntnisses aus unserer alten Heimat über den Ozean getragen und hier ausgesät“" (Kratochwil 2013: 187). Es zeigt sich aber bald, dass die Naturverbundenheit Holds moralisch untergraben ist. Seine zweite Frau Marta, eine Chilenin, hat er anscheinend die Treppe hinuntergestoßen. Er will sie beseitigen, um seine sexuellen Phantasien freier ausüben zu können, denn die erwähnte Cintia will er sich völlig unterwerfen. Gegen den Landankauf von Meyllonets Umweltplänen, um die Trinkwasserreserven vor einem chilenischen Staudammprojekt zu schützen, wehrt er sich scheinbar idealistisch, doch ist es möglich, dass eigene Profitgier dabei eine Rolle spielt. Als sich Marta in seiner Abwesenheit davon macht, fällt Holds Ausstieg völlig auseinander. Er glaubt, Marthas taubstummer Sohn stecke hinter den Plänen der Mutter, sucht ihn wütend auf, wobei dieser Hold aus Rache für die Mutter erschießt. Die Leiche wird in der Laguna Negra versenkt, was Kainzer, immer noch auf der Suche nach Melan, mit großem Schrecken beobachtet. Für Hold ist der Ausstieg ebenfalls gescheitert.

Über dem ganzen gewalttätigen Geschehen thront derweilen der amerikanische Kapitalist und Millionär Meyllonet, Don Tim genannt. ${ }^{9}$ Seine Ladenkette in den USA hat er im Zuge einer Scheidung gewinnträchtig absetzen können, baut hoch

$9 \quad$ Hinter dieser Figur steckt Douglas Tompkins (1943-2015), der Begründer von Esprit und North Face, der Millionen von Dollar in Nationalparks in Argentinien und Chile investierte. Vgl. Diana Saverin, „The Entrepreneur 
oben in Patagonien im Quellgebiet des Río Glacier eine Art Schloss und kauft massenweise das Land auf, um die Trinkwasserreserven vor staatlicher und privater Raubgier zu schützen. Dabei scharrt er, mit Ausnahme Holds, die lokale Bevölkerung um sich: „Don Tim cumple!, er hält seine Versprechen [...]. Er verspreche der Menschheit nicht nur das Heil, sondern versuche selbst, es ihr zu sichern. Und das Lebensheil sei nun einmal im reinen Wasser zu finden. Die Urquelle des Wohlbefindens ist Trinkwasser - für die Menschen, für die Ochsen, für die Pferde, für alle Lebewesen auf dem Planeten“ (Kratochwil 2013: 160). Er bleibt dabei „unverändert profitabel“ (Kratochwil 2013: 252), doch scheint er in der Tat ein „Wohltäter“ (Kratochwil 2013: 155) zu sein, der das 2003 von der UNO ausgerufene „Jahr des Süßwassers" ernst nimmt. ${ }^{10}$ Tatkräftig wird Meyllonet von seiner dreißig Jahre jüngeren, japanischen Frau Miyoko unterstützt. Ein Ausstieg scheint Meyllonet gelungen zu sein, der gesellschaftlich engagiert handfeste Ziele hat, im Gegensatz zur mystischen Verschwommenheit Melans oder zur moralischen Korruptheit Holds. Den Ausstieg hat sich Meyllonet zwar erkauft, doch nicht als rücksichtsloser Kapitalist, sondern vielmehr mit der Absicht, etwas Gutes mit seinem Geld machen zu wollen. In seiner Kritik des Romans meint Jan Koneffke, „[d] as reiche Weltenretterehepaar“ sei „dem Autor vielleicht doch ein wenig zu platt geraten“ (Koneffke 2013), d.h. im Gegensatz zur ansonsten ironisierenden Abrechnung des Romans mit Aussteigern. Der angebliche Mangel an Ironie mag aber auch damit zusammenhängen, dass Kratochwil dieses Ehepaar einfach ernster nimmt.

Kainzers Reise nach Patagonien ist zunächst einmal nicht von Aussteigergedanken motiviert. Doch indem er sich ins ferne Patagonien auf der Suche nach Livias Ehemann begibt, gerät seine Welt zunehmend in Unordnung, vor allem dadurch, dass er in das gewalttätige Geschehen um Hold und seine Frau hineingezogen wird. Kainzer setzt sich nach Puerto Montt ab, wo sich aber auch für ihn ein Ausstieg aus seinen bisherigen Verhältnissen andeutet. Hautnah bekommt er Proteste gegen das chilenische Staudammprojekt mit, die ihn zum engagierten Schreiben à la Egon Kisch anregen. Doch diese „Vision“ geht in ein „Trugbild“ (Kratochwil 2013: 293) über, wenn er sich überlegt, dass die erforderliche Präsenz in der Öffentlichkeit ihn mit dem Mord an Hold in Verbindung bringen könnte. Hinzu kommt die zufällige Begegnung mit Maia Morelli im Hotel, wo sie auf Melan wartet, der aber wegen seiner Verletzungen nie erscheint. Ihre Niedergeschlagenheit nützt er aus, um sie zu verführen. $\mathrm{Ob}$ es eine längerfristige Beziehung werden könnte, bleibt offen. Klarheit hat Kainzer jedenfalls nicht:

„Vollzogen und noch fragwürdiger geworden“, ging es ihm durch den Kopf; er wusste nicht, woher ihn das ansprach, woran er diesen allgemeinen Satz heften konnte. „Ich bin wirklich durcheinandergeraten.“ Wie hatte der Honorarkonsul in Villa Angostura den Freund [Melan] zitiert: „Im So-Sein der Erscheinung verbirgt sich die Chiffre ihrer Erklärung“"? (Kratochwil 2013: 301).

Wie schon im Scherbengericht wird auch hier deutlich, dass das Leben nicht groß mit einem abstrakten Erklärungsmodell zu erfassen, sondern sich scheinbar willkür-

Who Wants to Save Paradise“, The Atlantic (15.8.2014), http://www.theatlantic.com/business/archive/2014/09/ the-entrepreneur-who-wants-to-save-paradise/380116/. [01.04.2018].

10 Zum International Year of Freshwater vgl. etwa http://www.un.org/events/water/brochure.htm [01.04.2018]. 
lich, unberechenbar und chaotisch aus seinen Äußerlichkeiten ergibt. Kainzer blickt aus dem Hotelfenster: „Unendlich langsam, wie üblich zu dieser Jahreszeit, war die Astralnacht herabgesunken. In der Ferne, jenseits der auslaufenden Lichtpunkte entlang der Bucht, herrschte nun endgültig Finsternis. Der Journalist musste es ausformulieren: „Ein nachtschwarzes Aquarell'“ (Kratochwil 2013: 301). Es gibt keine Sicht, auch nicht in die Zukunft. Das metaphorische Bild bestätigt den Mangel an Zukunftsklarheit, die sich insgesamt auf Kratochwils Romanwelt übertragen lässt.

Die Kritik hat schon mehrfach auf die Schwächen des Romans hingewiesen, besonders im Vergleich zum Scherbengericht. Christoph Ohrem meint, Río Puro verrate zwar „den hohen Bildungsgrad seines Autors“, sei aber auch „stellenweise überambitioniert" (Ohrem 2013). Thorston Schulte schreibt, die Charaktere würden „unscharf" bleiben; der Autor versteige sich in „Nebenschauplätzen, ohne eine logische Einbindung in die Handlung zu erreichen" (Schulte 2014). Sein abschließendes Urteil lautet: „Wortverliebt ohne Angst vor derben Ausdrücken und mit voyeuristischem Blick auf das Treiben in den unwegsamen Tälern und entlegenen Orten entfaltet sich in ,Río Puro' ein wechselhaftes Schauspiel" (Schulte 2014). In der Tat ist Scherbengericht knapper und zusammenhängender erzählt; sein Griff über den Schauplatz Patagoniens hinaus bindet die Geschichte eines ganzen Jahrhunderts abwechslungsreicher in den Text hinein.

Dennoch kann Río Puro in mancher Hinsicht auch für sich stehen. Ausstiegsversuche von Menschen, die von einem Zurück-zur-Natur-Geist beseelt sind, das Wesentliche des Seins finden wollen oder sich ansonsten der Gesellschaft verweigern, hat eine lange Tradition in der Literatur. Miquel de Cervantes' Don Quijote de la Mancha (1605), Joseph von Eichendorfs Aus dem Leben eines Taugenichts (1826), Henry David Thoreaus Walden (1854), Hermann Hesses Steppenwolf (1927) oder J.D. Salingers Catcher in the Rye (1951) sind nur einige Beispiele. Lässt man die Aussteigerfiguren in Río Puro Revue passieren, scheinen sie durch erotische Altmännerphantasien motiviert zu sein. Ob man Melan, Hold, Meyllonet oder Kainzer näher betrachtet, jeder von ihnen sucht oder hat das erotische Abenteuer mit einer wesentlich jüngeren Frau gefunden. Doch belässt es Kratochwil nicht dabei, indem er diese Phantasien z.T. durch den Verlauf der Romanereignisse ironisiert oder auch eindeutig dekonstruiert, so im Falle von Melan und Hold. Jan Koneffke spricht sogar von ,[t]riefende[r] Ironie“ und vom „Grauen“ vor einigen Figuren (Koneffke 2013).

Kratochwil versieht auch Río Puro mit einem Stifter-Zitat, diesmal aus dessen Roman Der Condor (1840): ,[...] und fern, in den Urgebirgen der Cordilleren, wandelte ein unbekannter, starker, verachtender Mensch, um dort neue Himmel für sein wallendes, schaffendes, dürstendes, schuldlos gebliebenes Herz zu suchen" (Kratochwil 2013: 7). Wie ebenfalls im Scherbengericht steht dieses Zitat in einem ironischen Kontrast zum Geschehen um die Romanfiguren. Auch der „Río Puro“ des Romantitels ist ironisch zu verstehen, wenn er als Chiffre für einen reinen Idealzustand in der Natur stehen soll, dem aber die Romanhandlung als Möglichkeit mit der Ausnahme Meyollnets widerspricht. Zu unwägbar bleibt das Leben, als dass ideale, verschwommene Lebensvorstellungen ohne Haken umzusetzen wären, geschweige denn als Alternativen zu der verfahrenen Jahrhundertsituation in Scherbengericht. Dieses postmoderne Lebensgefühl breitet uns Kratochwil weiterhin unerschütterlich in Río Puro aus. 


\section{Territorium: Einbruch der großen Welt in Patagonien}

Territorium als Kratochwils letzter Roman der Trilogie ist am stärksten in unsere Gegenwart eingebunden. Im „Dank“ lesen wir vom Autor: „Mit Verblüffung habe ich erlebt, dass mir die , wirkliche Welt' beim Verfassen meiner Fiktion nachgelaufen ist, mich eingeholt und überholt hat“" (Kratochwil 2016: 326). Um welche „wirkliche Welt" es sich dreht, wird dann deutlich, als der 77-jährige Protagonist Eduard Böhm, ein ehemaliger Entwicklungshilfesoziologe und sicher wieder eine Alter-Ego-Figur des Autors, gerade den Fernseher einschaltet:

Mehrere Sender berichteten von neuen Gräueln des Islamischen Staates, von Krawallen der Nordafrikaner in Paris, von Brandstiftung in Asylbewerberheimen in Hessen, von Migrantenleichen an türkischen und griechischen Stränden, vom mysteriösen Tod des argentinischen Staatsanwalts Alberto Nisman (Mord oder Selbstmord?), von Waldbränden und Brandstiftern in Patagonien, von antisemitischer Hetze im bisher unauffälligen Quemquemtréu (Kratochwil 2016: 317).

Mit dem Übergang von realen Ereignissen, die ohne weiteres in der Presse aus den Jahren 2015-2016 nachgelesen werden können, zu den fiktionalen in Patagonien wären wir wieder am Schauplatz von Kratochwils Romanwelt in Quemquemtréu gelandet. Dort erscheint am Romananfang an der weiß gestrichenen Mauer des Yabrud Markets die militante Parole „TERRITORIUM UND FREIHEIT FÜR PALÄSTINA!“" Kratochwil 2016: 18), darunter die Abbildung eines AK-47, was schnell übermalt wird. Am Romanende kommt es dann zu einem Überfall „,mit Gewalt und wilden antisemistischen Parolen“ (Kratochwil 2016: 279) auf ein von jungen israelischen Touristen bewohntes Hostal.

Unheil verschiedenster Formen scheint in die patagonische Idylle einzubrechen, die die Jüdin Shira, aus Israel angereist, um ihren ebenfalls jüdischen, nach Argentinien ausgewanderten Ethan zu heiraten, wie folgt beschreibt: „Wie unterschiedlich all diese Gesichter - braun oder rosig in vielen Schattierungen -, die Haare blond, braun, schwarz, die Augen meist dunkel, seltener hell, rund oder nahezu ostasiatisch geschlitzt" (Kratochwil 2016: 60). Doch wird die von Einwanderung gekennzeichnete Rassenharmonie Patagoniens durch die militante Parole und den antisemitischen Überfall bedrohlich in Frage gestellt, vielleicht ein „Paradise lost“ (Kratochwil 2016: 304), wie es bei Böhm in Anspielung auf Milton heißt. Die hundertjährige Schamanin und Mapuche Kilakina Aurora Tokikurá sagt Düsteres voraus: „Ha, tanzt nur, tanzt vor dem Großen Geist! Bettelt um euer Leben! Ruft ihn um Hilfe an ... bevor es zu spät ist" (Kratochwil 2016: 66). Als groß angelegte Metapher für das Unheil dient der drohende Ausbruch des Milliqueo, eines seit Jahrhunderten eingeschlafen geglaubten Vulkans. Deswegen ist auch die Seismologin Clara Shuman im Rahmen eines „Andean Projekts“ aus Kalifornien angereist.

Letztendlich geht es um das „Territorium“ des Romantitels. Die Elendskulturen verschiedenster Formen hätten, so Böhm, ,auf längere Sicht eine expansive, einnehmende Kraft" und würden „,in die Enklaven der führenden Minderheiten eindringen“ (Kratochwil 2016: 94), was sich auch auf Patagonien und den übermalten Spruch eines palästinensischen Territoriumsanspruchs bezieht. Der Deutsch-Argentinier Carl Gustav Werneck, der angereiste, etwa zehnjährige jüngere Freund Böhms und sich um Gegenwartsprobleme kümmernde Publizist, erklärt die Situation in Patago- 
nien noch weiter im Sinne der Globalisierung und der damit verbundenen internationalen Verflechtungen in Wirtschaft, Politik, Kultur, Umwelt und Kommunikation. Seine Ausführungen erinnern an die Konflikte in Río Puro:

Es soll so abgelegen, abgeschottet, weltfern sein und wird jetzt doch - und wie! - in den aktuellen Sog der Globalisierung geraten. Allein schon weil sich zweitausend Meter tief unter uns das zweitgrößte Schieferöllager der Welt ausbreitet - und weil ein Stück südwärts im Kontinentaleis unendliche Süßwasserreserven ruhen. Um dieses Territorium wird man noch ganz andere Kämpfe führen als einst mein Urahn um sein Stückchen Weideland (Kratochwil 2016: 106).

Francesco Napoletano, ein Designer italienischer Abstammung, kommt wiederum mit einer marxistischen Interpretation der Globalisierung daher. Finanzkapitalismus und internationale Monopole würden zum Bestreben nach „Gewinnmaximierung“ führen, die wiederum nur ,durch mediale Versklavung der Konsumenten und Stimmabgaben-Automaten tragbar"sei: ,Wer sich absetzen könne und noch zu Lebzeiten seine Individualität retten wolle, der sollte sich einen weit entlegenen Zufluchtsort suchen. In dieser überhandnehmenden Finanz- und Medien-Globalisierung - einer wachsenden Wüste, die alle zu Sandkörnern zerreibe - könne man sich nur in Oasen retten“ (Kratochwil 2016: 55-56). Eine solche Oase ist eben Patagonien, dessen Idylle aber durch die weltlichen Machtkonflikte in der Suche nach „Territorium“ bedroht wird. $^{11}$

Es ist die Folie, vor der sich die persönlichen Schicksale der Romanfiguren abspielen. Da geht es z.B. um friedliche Lebensalternativen, gewissermaßen wie in Rio Puro um Ausstiegsversuche, um „Archipele besserer Welten“ (Kratochwil 2016: 56) in den Worten Shiras, die hofft, fern vom konfliktreichen Israel ein Leben zusammen mit Ethan aufzubauen. So gibt es auch Ari Broda und seine Brüder, deren Vater Miguel, Jude und argentinischer Sozialist, sich einer Kibbuz-Kooperative in Israel verschrieben hatte, doch unzufrieden den Weg nach Patagonien fand. Dort bauten die Söhne das Hostel auf, das eigentlich jungen Israelis nach Militärdienst und Trauma als Herberge bei ihren Reisen durch Südamerika dienen sollte, doch nun überfallen wird. Oder da ist der zwei- bis dreimal ertragreichere biointensive Gartenanbau von Mauro Peretti, der als Modell für die Ernährung der ärmsten Weltbevölkerung dienen könnte und für den sich viele interessieren: „Das sind Helfer und Praktikanten, die aus aller Welt, aus Australien, Wales, Schweden, Brasilien zu uns kommen. Sie wollen sich mit dem biointensiven Farming vertraut machen, vielleicht können sie es später ihren heimatlichen Bedingungen anpassen“ (Kratochwil 2016: 163). Allerdings meint Böhm, der Versuch werde eher „marginal“" bleiben, „denn der Mensch ist auf territoriale Beherrschung, auf Macht und Zerstörung aus“" (Kratochwil 2016: 162).

Nicht unerheblichen narrativen Raum im Sinne einer Lebensalternative nimmt auch die Geschichte von Alena und Emir ein: sie eine junge israelische Übersetzerin auf der Suche nach Erholung vom Militärdienst, doch kommt es zur Begegnung mit Emir, dem syrischen Neffen von Nadim Obeid, an dessen Laden in

11 Im erwähnten „Dank“ beruft sich Kratochwil in diesem Zusammenhang auf die bekannte Soziologin Saskia Sassen und deren Theorien über Territorium und Globalisierung (Kratochwil 2016: 326). Vgl. z.B. Sassen (2006). 
Quemquemtréu die ominöse Parole erschienen war. Der Onkel hatte ihn aus dem mittelöstlichen Konfliktgebiet nach Patagonien geholt, nachdem er durch einen Schuss aus einem israelischen Hubschrauber an der Hüfte verletzt wurde und hinkt. Als Übersetzerin kannte Alena dessen Bruder, der ein Jahr in israelischer Haft gewesen war, im Zuge eines Gefangenenaustausches frei kam, doch bald darauf bei einem Angriff in Südlibanon starb. Feindlich stehen sich Alena und Emir anfangs gegenüber, doch auf dem neutralen Boden Patagoniens zeichnet sich eine Aussöhnung ab, wie Emir andeutet: „Alena sagt, Territorium braucht nicht Betttuch zu sein, an dem man nur herumzerrt; man kann sich darauf auch lieb haben" (Kratochwil 2016: 322). Böhm schwört die Idylle herauf, um die es in Patagonien gehen könnte: „Ansonsten geht es bei uns hier bunt und multikulti zu: Hippies, Aussteiger, Anhänger der Permakultur, Gurus, Gestrandete, ein friedliches Miteinander und Durcheinander (Kratochwil 2016: 200).

Im Zentrum des Romans steht letztendlich die persönliche Entwicklung Böhms. Ähnlich Martin und Melan in Scherbengericht und Rio Puro ist ihm als ehemaligem Entwicklungshelfer viel Skepsis an einer Welt gewachsen, die scheinbar keine rationalen Erklärungsversuche zulässt: „Er hielt längst nichts mehr von Gesamtsichten, glaubte nur noch an kurzlebige, geistig oder emotional erworbene Erkenntnisfetzen, vermutete den Widerspruch und die Widerlegung gleich um die nächste Ecke" (Kratochwil 2016: 147). Im Gespräch mit Clara lesen wir: „Ich werde nicht fertig mit der Welt, wie sie ist, und ich bin feig, verwirrt und habe es satt - und dazu kommen noch meine Jahre! Es bleibt mir immer weniger Zeit..." (Kratochwil 2016: 99). Als Zeichen seines unsicheren Schwebezustands mag Böhms zwanghafte Vorstellung sein, durch die Luft fliegen zu müssen. Um die Lebenskrise zu verarbeiten, schlägt ihm der Psychologe Elias Königsberg vor, den wir schon aus Scherbengericht kennen, zurückgezogen in Patagonien ein „Waldhüttenprotokoll“ (Kratochwil 2016: 13) zu schreiben. Das tut Böhm auch, was sozusagen zu einer narrativen Überraschung gerät, denn das 6. Kapitel „Vorsicht, wir verlassen festen Boden!“ (Kratochwil 2016: 249ff.) lesen wir zunächst als Fortführung der Handlung, die sich jedoch später als eben dieses Waldhüttenprotokoll entpuppt. Abgesehen von einer erotischen Begegnung mit Clara stellt sich Böhm darin ein Treffen zwischen Alena und Emir an einem See vor, bevor der Vulkan ausbricht, aus dessen Ascheschlamm sie sich retten und „ein menschenwürdiges Gehen auf zwei Beinen“ (Kratochwil 2016: 273) versuchen. Böhms Phantasie vereint eine Lebensalternative der überbrückenden Menschlichkeit mit dennoch nicht zu entkommendem Unheil.

Das Waldhüttenprotokoll mit seinem Schwebezustand zwischen Hoffnung und Katastrophe nimmt in einem gewissen Sinne die weitere Romanhandlung vorweg. Statt Vulkanausbruch kommt es wegen einer eventuell dem Klimawechsel verschuldeten Trockenheit zu einem Waldbrand, dem Böhms Hütte zum Opfer fällt, ganz abgesehen von dem anscheinend antisemitischen Überfall auf das Hostal. Doch bleiben die Ursachen unklar. Hinter dem Waldbrand könnten auch korrupte Spekulationen stecken, um das beschädigte Land billig abzustoßen und den Gewinn in unbekannte Taschen fließen zu lassen. Der Überfall muss auch nicht unbedingt antisemitisch motiviert sein. Im Gespräch mit Nadim meint Böhm: „Gewiss, aber andere knüpfen Fäden wie für ein Spinnennetz, verbinden die Interessen des einen mit denen des anderen, und schließlich kommt es zu einem Verbrechen“"(Kratochwil 2016: 308). Werneck ist schon längst abgereist, aber aus der Ferne Deutschlands spricht er noch einmal von einem ,unaufhaltsam überall vordringenden Konfliktpo- 
tenzial in der Globalität" und von ,riskanten Randphänomenen der Globalisierung“ (Kratochwil 2016: 312).

Die Romanereignisse wirken sich auf die persönliche Entwicklung Böhms aus:

In diesem Dämmerzustand war ihm nicht mehr klar, wie viel in ihm zerstört worden war, wie das mit dem Pogrom zusammenhing, mit dem Hüttenbrand und mit dem Verlust der Vogelstimmen und des klaren Morgenlichts über dem Tal vor seiner Tür - verloren für immer ... Nur eine Meeresküste und Claras Umarmung könnten mich retten (Kratochwil 2016: 298).

In der letzten Instanz ist aber wie schon bei Kainzer in Rio Puro keine Klarheit zu schaffen. Ein neuer Lebensanfang mit Clara, schon wieder zu Hause in Kalifornien, ist nicht realistisch. An der Meeresküste ist vielleicht Unterschlupf zu finden, vielleicht ,ein richtiges Haus, ganz aus Granit, in Bahía Bustamente [...] hoch über der Steilküste, mit Blick auf den Atlantik? Dort wächst kein Baum“" (Kratochwil 2016: 301). Ohnehin gibt die Entwicklung eines Slums am Rande von Quemquemtréu Böhm den Rest: „So etwas hatte es in der ganzen Region noch nie gegeben - das war genau das Ambiente, dem der Slum-Forscher Eduard Böhm während der Jahrzehnte seines Berufslebens ständig zu entfliehen träumte. ,That's it', stieß er laut hervor [...]“ (Kratochwil 2016: 321). Was von der abgebrannten Waldhütte bleibt, wird notdürftig gerettet, womit der Roman ausklingt: „Dann galt es, den Ort zu verlassen“ (Kratochwil 2016: 325). Ob das bedeutet, auch Quemquemtréu zu verlassen, bleibt in der Schwebe. Die mögliche Alternative des Granithauses an der Atlantikküste hört sich ohnehin wie ein Einbunkern angesichts einer bedrohlichen Realität an, mit der Böhm nur schlecht zurecht kommt.

Die Romanwelt mit ihren vielen Figuren, die Kratochwil in Territorium schafft, ist zu reichhaltig, als dass sie sich hier adäquat einfangen ließe. Vielleicht sind es letztendlich auch zu viele Stimmen, die der Autor unterzubringen versucht, abgesehen von romantisch-erotischen Abschweifungen, die wenig zur Handlung beitragen (vgl. Anonym 2016). Doch die Grundfrage des Romans bleibt relevant. Zwar wird das militante Graffiti am Romananfang schnell übermalt, doch lässt sich dadurch der Einbruch der Globalisierung und des Nahostkonflikts in die patagonische Idylle verhindern? (vgl. Widmann 2016). Die Frage wird nicht eindeutig beantwortet, woraus sich wieder der für Kratochwil charakteristische Schwebezustand seiner Romane ergibt. Von den großen Weltkonflikten können wir in den Medien täglich lesen, wo sie eventuell abstrakt bleiben. In Territorium und anhand des patagonischen Schauplatzes führt uns der Autor vor, wie sie sich auch im Kleinen und Persönlichen auswirken könnten.

\section{Schlussbetrachtung}

In Krachtowils patagonischer Romantrilogie führen Auseinandersetzungen mit Vergangenheit und Gegenwart, widersprüchliche Ausstiegsversuche und die Auswirkungen globaler Konflikte zu einem zunehmend ungesicherten Weltbild, das als wunderlich, verworren, absurd, bizarre, grotesk und zufällig bezeichnet werden kann. Dem sich daraus ergebenden Schwebezustand der Ungewissheit entkommt man auch nicht im fernen Patagonien oder überhaupt in irgendeiner isolierten Welte- 
cke. Wohin soll das alles führen? Kratochwil gibt uns keine fertigen Antworten. Doch ist es letztendlich eine ziemlich düstere Vision unserer Zukunft, die sich bestenfalls ziellos und ohne scheinbare (Er-)Lösungen fortsetzen wird.

\section{Literaturverzeichnis}

Anonym, «Neue Bücher - German Kratochwil: ,Territorium’ verdrängt das Paradies»,

Tiroler Tageszeitung (30.8.2016), http://www.tt.com/home/11952799-91/neue-bücher---german-kratochwil-territorium-verdrängt-das-paradies.csp. [01.04.2018].

Drekonja-Kornat, G., «Für eine halbe Million Dollar. Stürmisch: Germán Kratochwils Odyssee durch Patagonien», Die Presse (20.9.2013), http://diepresse.com/home/spectrum/literatur/1455354/Fur-eine-halbe-Million-Dollar. [01.04.2018].

Knipphals, D., «Gestandet in Patagonien», taz (26.5.2012), http://www.taz.de/1/archiv/digitaz/artikel/?ressort $=$ ku\&dig $=2012 \% 2 \mathrm{~F} 05 \% 2 \mathrm{~F} 26 \% 2 \mathrm{Fa} 0047 \% \mathrm{cHash}=400811 \mathrm{a} 4 \mathrm{f} 8$. [01.04.2018].

Koneffke, J., «Natur und Müll», Neue Zürcher Zeitung (23.11.2013), http://www.nzz.ch/aktuell/feuilleton/literatur/natur-und-muell-1.18190540. [01.04.2018].

Kratochwil, G., Río Puro. Roman. Wien: Picus 2013.

Kratochwil, G., Scherbengericht. Roman. Wien: Picus 2012.

Kratochwil, G., Territorium. Roman. Wien: Picus Verlag 2016.

Ohrem, C., «Mit einer halben Million in den Anden verschollen», Westdeutscher Rundfunk (30.10.2013), http://www.wdr3.de/literatur/riopuro104.html. [01.04.2018].

Sassen, S.: Territory - Authority - Rights. From Medieval to Global Assemblages. Princeton: Princeton University Press 2006.

Schönherr, V., «Scherben, aber kein Gericht», WOZ Die Wochenzeitung (14.2.2013), https:// www.woz.ch/1307/argentinien-und-die-nazis/scherben-aber-kein-geeicht. [01.04.2018].

Schulte, T., «Trübe Gefühle und Langeweile in der Wildnis. Germán Kratochwils Roman ,Río puro' über skurrile Reiseabenteuer zweier Männer in Patagonien», Literaturkritik (Januar 2014), http://www.literaturkritik.de/public/druckfassung_rez.php?rez_id=18738. [01.04.2018]

Widmann, C., «Albtraumpfade», Süddeutsche Zeitung (14.11.2016), http://www.sueddeutsche.de/kultur/belletristik-albtraumpfade-1.3248809. [01.04.2018]. 